\title{
Design and Implementation of the Symbol Table for Object- Oriented Programming Language
}

\author{
Yangsun Lee \\ Dept. of of Computer Engineering, Seokyeong University \\ 16-1 Jungneung-Dong, Sungbuk-Ku, Seoul 136-704, KOREA \\ yslee@skuniv.ac.kr
}

\begin{abstract}
The symbol table used in the existing compiler stores one symbol information into a plurality of sub tables, and the abstract syntax tree necessary for generating symbols has a binary tree structure composed of a single data structure node. This structure increases the source code complexity of modules that generate symbols and modules that reference symbol tables, and when designing a compiler for a new language, it is necessary to newly design an abstract syntax tree and a symbol table structure considering the characteristics of the language.

In this paper, we apply the object-oriented principle and visitor pattern to improve the abstract syntax tree structure and design and implement the symbol table for the object oriented language. The design of AST (abstract syntax trees) with object-oriented principles and Visitor patterns reduces the time and cost of redesign because it makes it easy to add features of the language without the need to redesign the AST (abstract syntax tree) for the new object-oriented language. In addition, it is easy to create a symbol through the Visitor pattern. Symbol tables using the open-close principle and the dependency inversion principle can improve the code reusability of the source code that creates and refer to the table and improve the readability of the code.
\end{abstract}

Keywords: Symbol Table, Compiler, Object-oriented Principle, Visitor Pattern, AST

\section{Introduction}

A compiler is a system software that translates user-written programs into machinereadable instructions that can be executed by a computer. Because of the nature of the compiler, the development of a new compiler is required whenever the language and the platform are different, various studies have been conducted on compiler modularization. Despite these researches, however, the symbol table for storing and managing symbol information was made up of a number of sub-tables and was a complicated structure referring to each other. In addition, since the abstract syntax tree used to represent the characteristics of the language and generate symbols is a binary tree structure representing all kinds of nodes with a single data structure, it is necessary to change the overall structure of the tree when a new language feature is added, There has been a problem that a whole modification of the routines using the tree is necessary. Symbol generation process, which collects and completes symbol information, also analyzes the abstract syntax tree through complex conditional statements and recursive calls, so the complexity of the compiler source code is still high and maintainability is poor [1,2].

In order to solve this problem in this paper, we have improved the structure of the abstract syntax tree by applying the object-oriented principle and Visitor pattern to easily maintain the characteristics of the existing object-oriented language and to easily add new characteristics. In order to reduce the complexity of reference routines, we designed and implemented a symbol table based on object-oriented principles. Improved abstract syntax trees make it easy to add properties without modifying existing tree structures when 
creating a tree for a language of the same object-oriented language. The process of generating the symbol using the object-oriented principle traverses the abstract syntax tree node through the Visitor class, performs semantic analysis to collect symbol information without additional conditional statements, and inserts the symbol information into the symbol table. Since it is expressed by only two tables, it is possible to reduce the source code complexity of the process of generating a symbol and the process of referring to a symbol table.

\section{Researches}

\subsection{AST (Abstract Syntax Tree)}

The compiler expresses the program in a tree structure in order to express the meaning of the input program more effectively, and it is easily reconfigured according to the characteristic of the code. Parse tree is one of these tree representation methods. However, because the parse tree contains a lot of unnecessary redundant information, it is inappropriate for intermediate languages and requires a more efficient method. Therefore, the abstract syntax tree (AST) is an expression method that removes unnecessary information from the parse tree. The AST node is divided into a terminal node and a nonterminal node. A terminal node represents a constant or a variable, and a non-terminal node represents an AST operator. The operands of one operator are bound to child nodes and are organized in a subtree form with their operator nodes. Figure 1 shows the Figure 1 shows an example of an AST for the integer type variable $\mathrm{x}$.

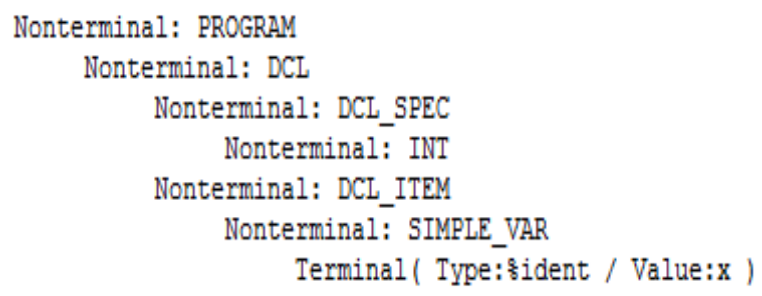

Figure 1. Example of an AST for the Integer Type Variable $x$

\subsection{Visitor Pattern}

Visitor patterns, one of the software design patterns, allow you to add new behavior to the object structure without modifying the existing structure by separating the structure and function of the object. It is designed in such a way that a separate visitor class is created by collecting the functions of each class and each object is circulated by the visitor class. The visitor pattern is suitable for cases where the structure of the object does not change well, and is also suitable when additional functions are required in the future. Regardless of the type or structure of the object, you can use the Visitor object to perform the function anywhere you have an interface for the visit. Figure 2 shows the Visitor class of the visitor pattern visiting each element. 


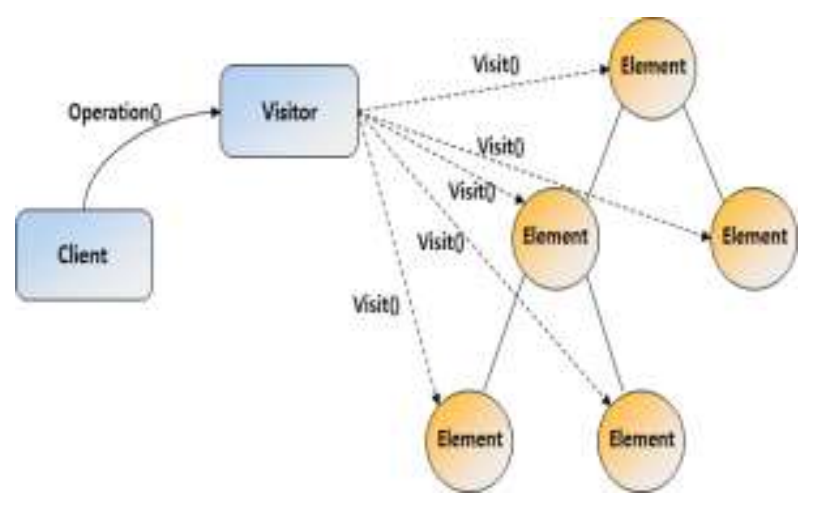

Figure 2. Visitor Pattern Visiting Each Element

\subsection{Code Metrics}

A code metrics is a measure of the quality of source code quality. It includes maintenance index, class linkage, inheritance level, number of lines of code, and halstead metrics. A maintenance index is a measure of the relative ease of maintenance of a code with a value between 0 and 100 . Numerical values are calculated using the number of lines of code, program volume, and cyclic complexity. The larger the value, the more convenient the maintenance work. The color value can be set according to the numerical value, and the ease of maintenance of the code can be visually judged.

Class coupling metrics measure the degree of association of classes by parameters in a class, local variables, return types, method invocations, and interface implementations. The lower the degree of class coupling, the higher the cohesion, which means that the higher the degree of coupling, the more difficult it is to maintain the source code. Inheritance level refers to the number of class definitions that extend to the root in the class hierarchy. The more complex the hierarchy, the more difficult it is to determine where certain methods and fields are defined or redefined.

The number of lines of code is a measure of the number of lines in the source code, which tells you how much work is done on the method, and the higher the number, the more difficult it is to maintain. Circular complexity is a metric developed by McCabe in 1976 that measures the complexity by the number of paths based on a control flow graph that represents the logic flow of the program. It is easy to compare different programs by measuring the complexity of the source code and presenting the result as a single value. Numbers are calculated based on the number of control statements.

Halstead metrics is an index of quantitative complexity based on the number of operators and operands in source code. It was introduced by Maurice Howard Halstead in 1977. Halstead metrics is a program that measures the program length, the number of program vocabulary, the program volume, the program's level of understanding of the program and its maintainability, and the effort required to understand and implement the program code. , And method and type, and the number of estimated bugs, which is the estimated value of the number of bugs that can occur in the package.

To compare the analysis and improvement of the symbol table structure to which the object-oriented principle is applied, a code metric based on the control flow of the code is required. Typical metrics include a halstead metrics and cyclic complexity. Halsted metrics can obtain maintenance-specific values that can't be calculated by cyclic complexity, but they are difficult to use in the program design stage because they are calculated based on the number of operators and operands. Also, unlike the cyclic complexity, which shows the complexity of the control flow, the control flow is not reflected, so the halstead metrics can be high even in source code without conditional statements. Therefore, we use the cyclomatic complexity based on control flow for analysis and evaluation of symbol table source code. 


\section{AST Structure Improvement}

The structure of the AST (Abstract Syntax Tree) node used in the existing compiler expresses information of all terminals and non-terminal nodes as one structure. In this structure, the analysis module using the AST can classify the node type through the attribute of the node, and the analysis process according to the type can be performed. Therefore, the maintenance complexity of the source code is increased and the maintainability is degraded. The complexity of the AST analysis process affects the symbol generation process because of the structure of the compiler that generates symbols through the analysis of AST. To solve this problem, we designed the node structure of AST as layered object structure according to object orientation principle.

The information of the AST node is represented as a class object. The hierarchical structure of the node is the highest class, and there exists the AST Node class and the nonterminal Node class and the terminal node class which inherit it. Figure 3 shows the hierarchy of the AST nodes.

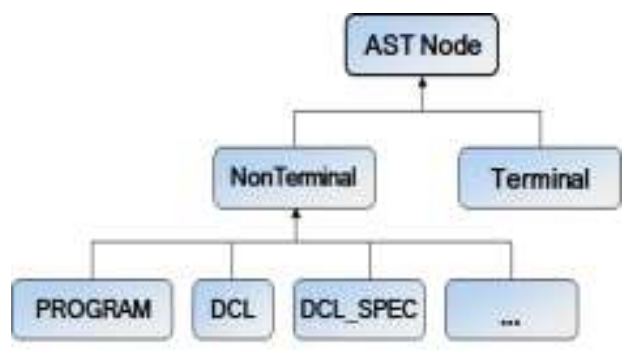

Figure 3. Hierarchy Structure of the AST Nodes

The terminal node class represents the terminal node information, and the node corresponding to the variable and constant information is defined by the corresponding class. The non-terminal node class classifies non-terminal nodes into child classes that represent information of non-terminal nodes. In this way, the node class classified according to each node information has a structure suitable to the principle of single responsibility. In addition, the AST Node class, which is the best parent class of a node, is defined as an abstract class so that it can observe the polymorphism and the liskov substitution principle in the semantic analysis step of analyzing the AST structure. The improvement of the AST structure does not require any additional cost since only the type of node generated in the same manner as the existing generation routine is changed into the hierarchical class without changing the generation routine.

Figure 4 shows an example of the layered node structure of the AST structure in Figure 2. The non-terminal node indicates the type of the non-terminal node by the name of the node, and the terminal node class indicates 'T:' meaning terminal, and the corresponding class is the terminal node class.

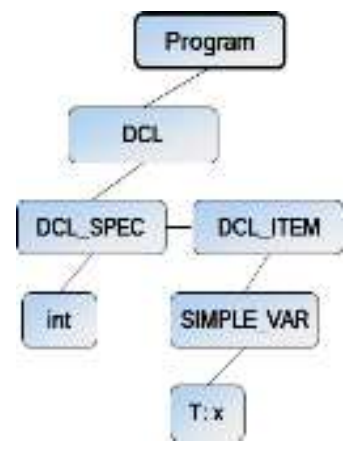

Figure 4. Layered Node Structure of the AST Structure 


\section{Symbol Table Design}

We design a symbol table structure using object-oriented principles and design a hierarchical symbol class to store symbols according to the symbol table to which this principle applies. In addition, a type class for storing the necessary type information in the symbol is designed and a type table for managing the type object is designed.

\subsection{Hierarchical Design of Symbols}

The symbol table stores hierarchical symbol objects to obtain symbol information with minimum conditions. The symbol class is designed as a hierarchical structure that has the abstract class Symbol as its top class and has Variable, Field, Function, Method, and Parameter as subclasses. Unlike the existing symbol table structure, which stores symbols in multiple tables, the symbol table to which the object-oriented principle is applied stores symbol attributes as one of the subclasses of the symbol class in the hierarchical structure.

Figure 5 shows the hierarchy of symbols. Each symbol subclass derived from Symbol class is a class for a node that represents the characteristics of a symbol among nonterminal nodes. The nodes corresponding to the variable information are subclasses inheriting the Variable class, and the nodes having the function and method information inherit the Function and Method classes.

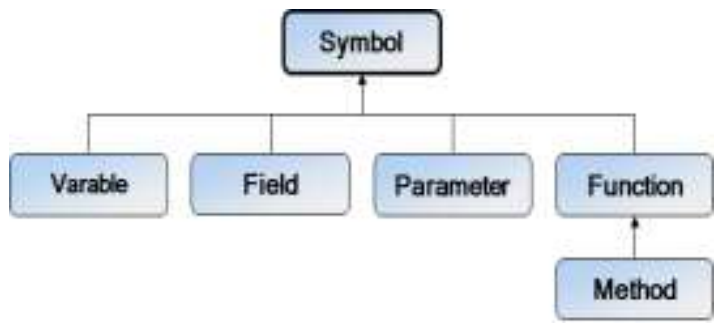

Figure 5. Hierarchical Symbol Table Structure

\subsection{Symbol Table Structure}

The symbol table stores the symbol object as a value with the symbol name as a key. The attributes of a symbol are stored in a symbol defined as a subclass type of a symbol hierarchy applying the object-oriented principle, and symbol type information is referenced in a type table. When referring to a symbol table from the outside, a symbol is searched based on the symbol name. Since it is made up of objects corresponding to each kind of symbols, additional analysis of symbols is not necessary. Figure 6 shows the structure in which the symbol table has symbol names and symbol values.
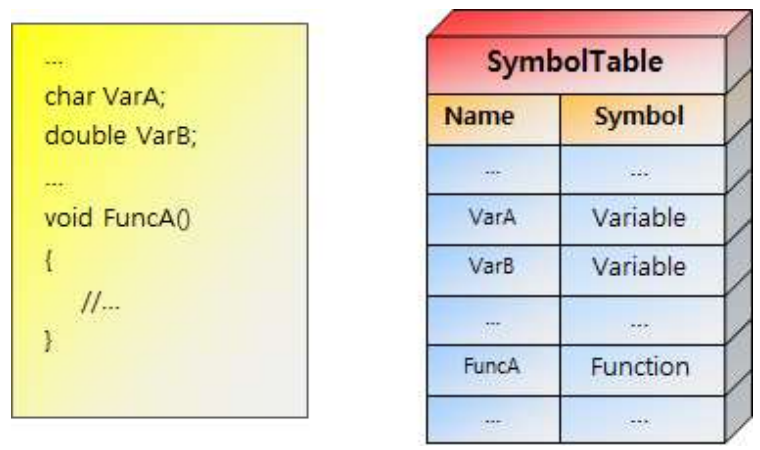

Figure 6. Symbol Table Structure 


\subsection{Hierarchical Design of Type}

The type table is a table for managing symbol type information. The type is designed as a hierarchical structure having the abstract class Type as the top class as the symbol. Primitive, which is a subclass of Type, is a general type class such as Int and Char. It is composed of Reference class, which is a reference type, and UserDefined class, which is a user defined type such as Typedef, Interface, and Struct. Figure 7 shows the type hierarchy.

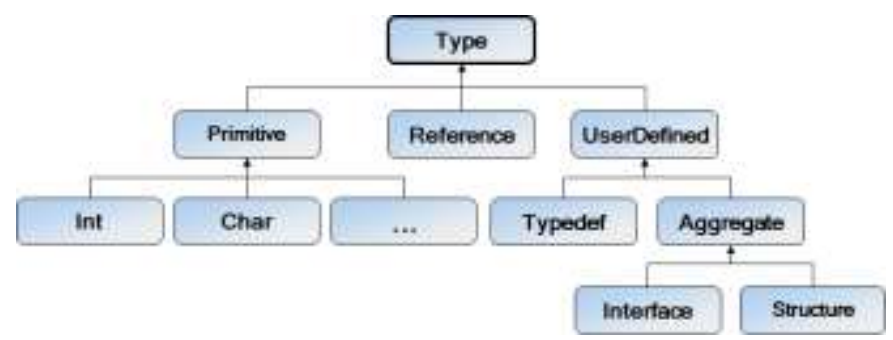

Figure 7. Type Hierarchy Structure

\subsection{Type Table Structure}

The type table stores the name of the type as a key and the type object as a value. The type attribute is stored in a hierarchical type, and the type information stored in the type table is referred to when referring to the symbol type. If multiple types exist in one type information, such as a typedef, the type information is linked and managed in a list form. Figure 8 shows the structure for storing basic type objects in a type table.

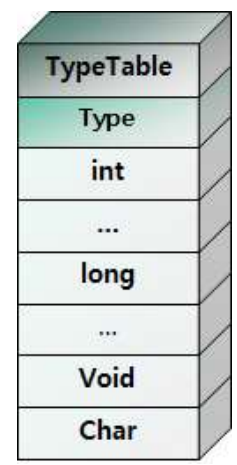

Figure 8. Type Table Structure

\subsection{Symbol Generator}

The symbol generator traverses the abstract syntax tree through the abstract syntax tree visitor class applying the visitor pattern structure, collects symbol information, inserts the symbol into the symbol table, and completes the symbol. Figure 9 shows the structure of the symbol generator.

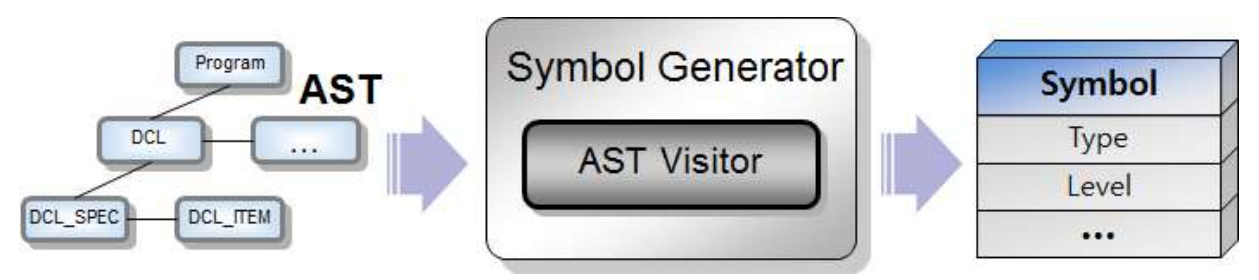

Figure 9. Symbol Generator Structure 
The structure of the abstract syntax tree visitor is divided into the visitor generation class that traverses the tree and the node visit, and the symbol generation process that collects the semantic analysis functions of the abstract syntax tree nodes. The function of abstract syntax tree visitor is divided into tree traversal and node visit. First, the tree traversal traverses the abstract syntax tree received as input. Next, the node visit visits the node that is encountered during the tree traversal, and performs the symbol generation process of the visited node through the visitor to collect symbol information from the node. Figure 10 shows how a visitor visits an abstract syntax tree node and processes the node.

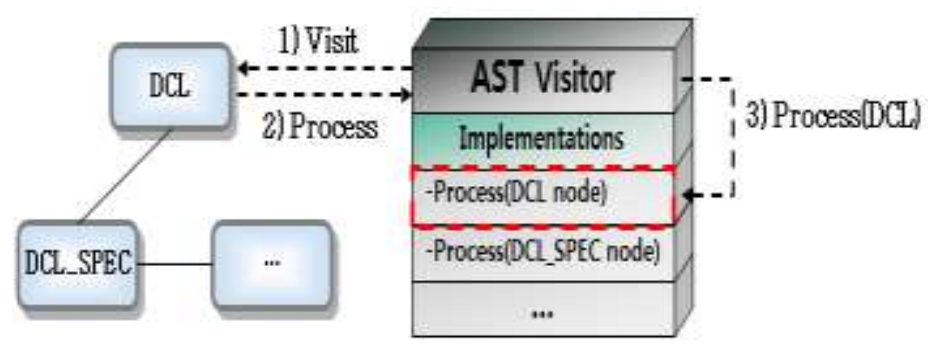

Figure 10. AST Node Processing through Visitor

The node visiting the abstract syntax tree traverses the symbol generation process through the visitor and collects the symbol information of each node. It collects information such as name, type, level, initial value and so on necessary to construct the symbol, and completes one symbol. The completed symbol is inserted into the symbol table.

\subsection{Structure of Symbol Table}

The symbol table structure using the proposed symbol table is as follows. Figure 11 shows the management structure of a single type symbol. In the symbol table, VarA, which is a variable name, acts as a key of a symbol table. A Variable object stored as a value refers to Char type, which is a type of VarA, in the type table, Const information indicating a constant type variable, whether or not to initialize, and the like are stored in the object.The type table handles the basic type as an object in the same way as the symbol table, and it shows the storing of Char and int type information used in VarA and VarB.

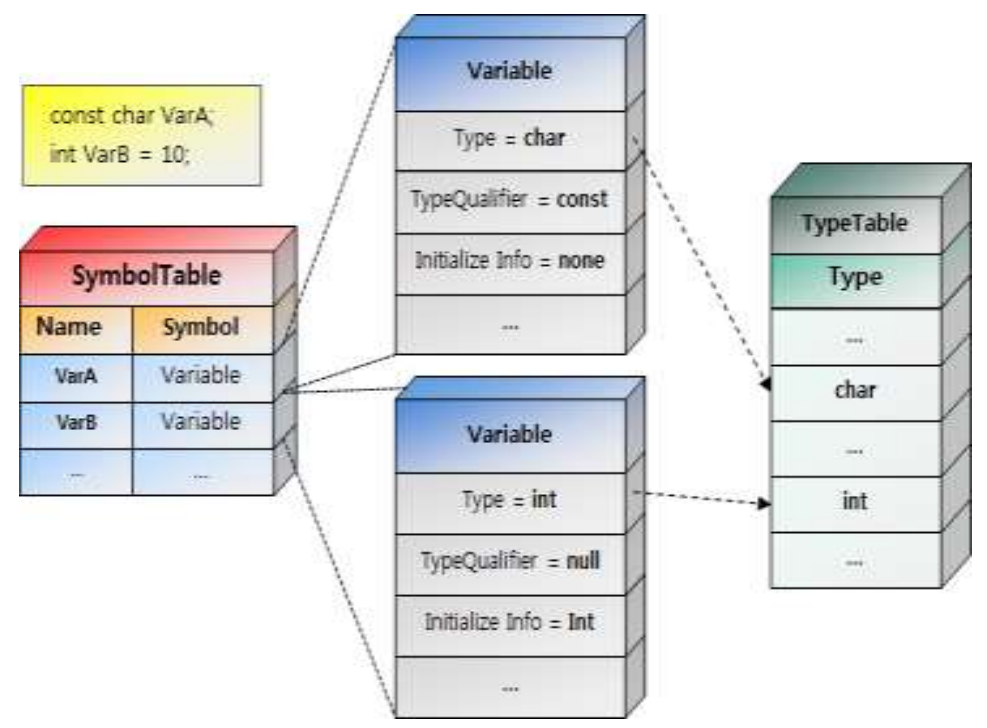

Figure 11. Management Structure of a Single Type Symbol 


\section{Experimental Results and Analysis}

First, we confirmed that a compiler applying the proposed AST structure for CPP, Objective $\mathrm{C}$, and Java language input programs having the same meaning normally generates AST. Example 1 shows the AST generation for the CPP source code, example 2 shows the AST generation for the Objective C source code, and finally example 3 shows the AST generation for the Java source code.

\begin{tabular}{|c|c|}
\hline CPP Source Code & CPP AST \\
\hline 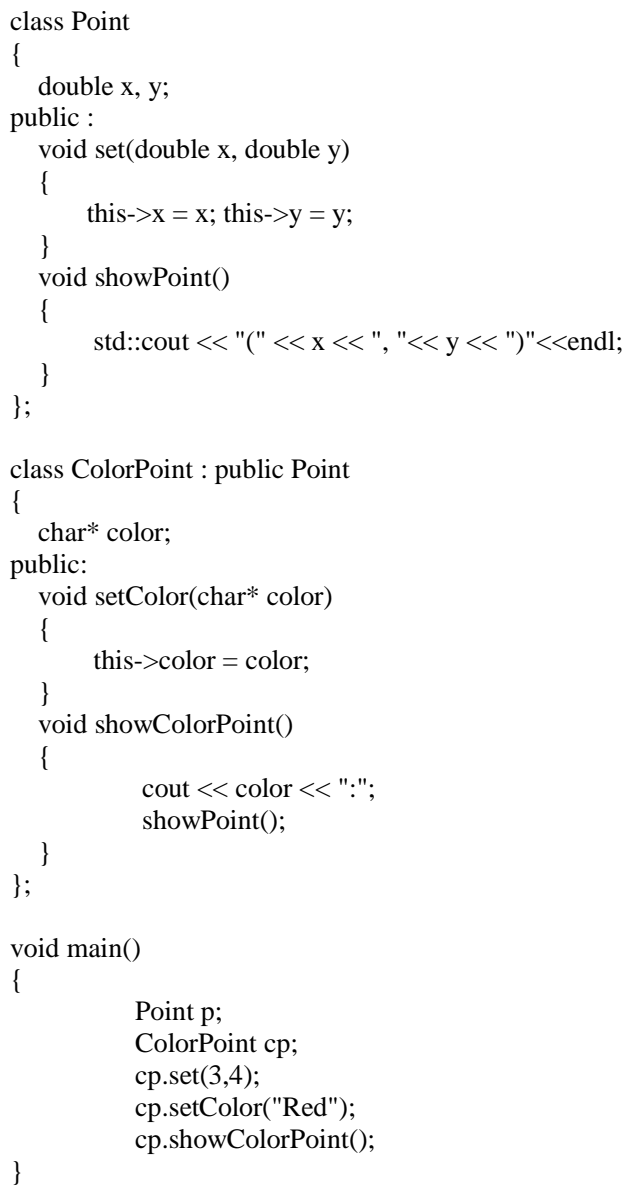 & $\begin{array}{l}\text { Nonterminal: PROGRAM } \\
\text { Nonterminal: DCL } \\
\text { Nonterminal: DCL_SPEC } \\
\text { Nonterminal: CLASS_DCL } \\
\text { Nonterminal: CLASS_HEAD } \\
\text { Terminal( Type:id / Value:Point ) } \\
\text { Nonterminal: MEMBER_DCL } \\
\text { Nonterminal: DCL_SEEC } \\
\text { Nonterminal: DOUBLE } \\
\text { Nonterminal: DCL_ITEM } \\
\text { Nonterminal: SIMPLE_VAR } \\
\text { Terminal( Type:id / Value:x ) } \\
\text { Nonterminal: DCL_ITEM } \\
\text { Nonterminal: SIMPLE_VAR } \\
\text { Terminal( Type:id / Value:y ) } \\
\text { Nonterminal: PUBBIC } \\
\text { Terminal( Type:tpublic / Value:public ) } \\
\text { Nonterminal: MEMBER_FUNC_DEF } \\
\text { ‥ } \\
\text { Nonterminal: FUNC_DEF } \\
\text { Nonterminal: DCL_SPEC } \\
\text { Nonterminal: INT } \\
\text { Nonterminal: FUNC_DCL } \\
\text { Nonterminal: SIMPLE_VAR } \\
\text { Terminal( Type:id / Value:main ) } \\
\text { Nonterminal: COMPOUND_ST } \\
\text { Nonterminal: DCL } \\
\text { Nonterminal: DCL_SPEC } \\
\text { Nonterminal: TYPE_NAME } \\
\text { Nonterminal: CLASS_TAG } \\
\text { Terminal( Type:className / Value:Point ) } \\
\text { Nonterminal: DCL_ITEM } \\
\text { Nonterminal: SIMPLE_VAR } \\
\text { Terminal( Type:id / Value:p ) } \\
\text {... }\end{array}$ \\
\hline
\end{tabular}

Example 1. AST Generation for the CPP Source Code

\begin{tabular}{|l|c|}
\hline \multicolumn{1}{|c|}{ Objective C Source Code } & Objective C AST \\
\hline //Point.h & Nonterminal: PROGRAM \\
@onterminal: CLASS_INTERFACE \\
@interface Point : NSObject & Terminal( Type:\%ident / Value:Point ) \\
@ property (readwrite) double_x; & Nonterminal: INTERFACE_DCL_LIST \\
@ property (readwrite) double_y; & Nonterminal: PROPERTY_DCL \\
-(void) showPoint; & Terminal( Type:@property / Value:@property ) \\
@end; & Nonterminal: PROPERTY_ATTRIBUTES \\
//point.m & Nonterminal: SIMPLE_PROPERTY_ATTRIBUTE \\
@implemantation Point & Terminal( Type:\%ident / Value:readwrite ) \\
@ synthesize_x; & Nonterminal: DCL \\
@synthesize_y; & Nonterminal: DCL_SPEC \\
-(void) showPoint & Nonterminal: DOUBLE \\
\{ & Nonterminal: DCL_ITEM \\
& Nonterminal: SIMPLE_VAR \\
& Terminal( Type:\%ident / Value:_x ) \\
\hline
\end{tabular}




\begin{tabular}{|c|c|}
\hline \multirow{2}{*}{\multicolumn{2}{|c|}{ NSLog(@"(\%lf, \%lf)\n",_x,_y); }} \\
\hline & \\
\hline @end & Nonterminal: DCL_SPEC \\
\hline & Nonterminal: INT \\
\hline //ColorPoint.h & $\begin{array}{l}\text { Nonterminal: FUNC_DCL } \\
\text { Nonterminal-SIMPLE VAR }\end{array}$ \\
\hline @interface ColorPoint : Point & 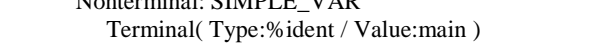 \\
\hline @ property (copy) NSString*_color; & Nonterminal: PARAM_DCL \\
\hline -(void) showColorPoint; & Nonterminal: INT \\
\hline @end & $\begin{array}{l}\text { Nonterminal: SIMPLE_VAR } \\
\text { Terminal( Type:\%ident / Value:argc ) }\end{array}$ \\
\hline //ColorPoint.m & Nonterminal: PARAM_DCL \\
\hline @implemantation ColorPoint & Nonterminal: CONST \\
\hline @ synthesize_color; & Nonterminal: CHAR \\
\hline -(void) showColorPoint & Nonterminal: POINTER \\
\hline & Nonterminal: SIMPLE_E_VAR \\
\hline NSLog(@"\%@ :",_color); & Terminal( Type:\%ident / Value:argv ) \\
\hline [super showPoint]; & $\begin{array}{l}\text { Nonterminal: COMPUND_ST } \\
\text { Nonterminal: DCL }\end{array}$ \\
\hline @end & Nonterminal: DCL_SPEC \\
\hline (®end & Nonterminal: CL̄ASS \\
\hline //main.m & Terminal( Type:\%class_name / Value:Point ) \\
\hline void main (int $\operatorname{argc}$, const char $* \operatorname{argv}[])$ & $\begin{array}{l}\text { Nonterminal: DCL_MEM } \\
\text { Nonterminal: POINTER }\end{array}$ \\
\hline \{ & Nonterminal: SIMPLE_VAR \\
\hline Point $* \mathrm{p}=[[$ Point alloc $]$ init $]$; & Terminal( Type:\%ident / Value:p ) \\
\hline & Nonterminal: MESSAGE_EXP \\
\hline ColorPoint $* \mathrm{cp}=[[$ ColorPoint alloc $]$ init $]$; & Nonterminal: RECEIVER_PART \\
\hline cp._x $=3.1$ & $\begin{array}{l}\text { Nonterminal: MESSAGE_EXP } \\
\text { Nonterminal: RECEIVER PART }\end{array}$ \\
\hline cp._y = 4.5; & \\
\hline cp._color = @ "Black"; & \\
\hline [cp showColorPoint]; & \\
\hline$\}$ & \\
\hline
\end{tabular}

\section{Example 2. AST Generation for the Objective C Source Code}

\begin{tabular}{|c|c|}
\hline Java Source Code & Java AST \\
\hline 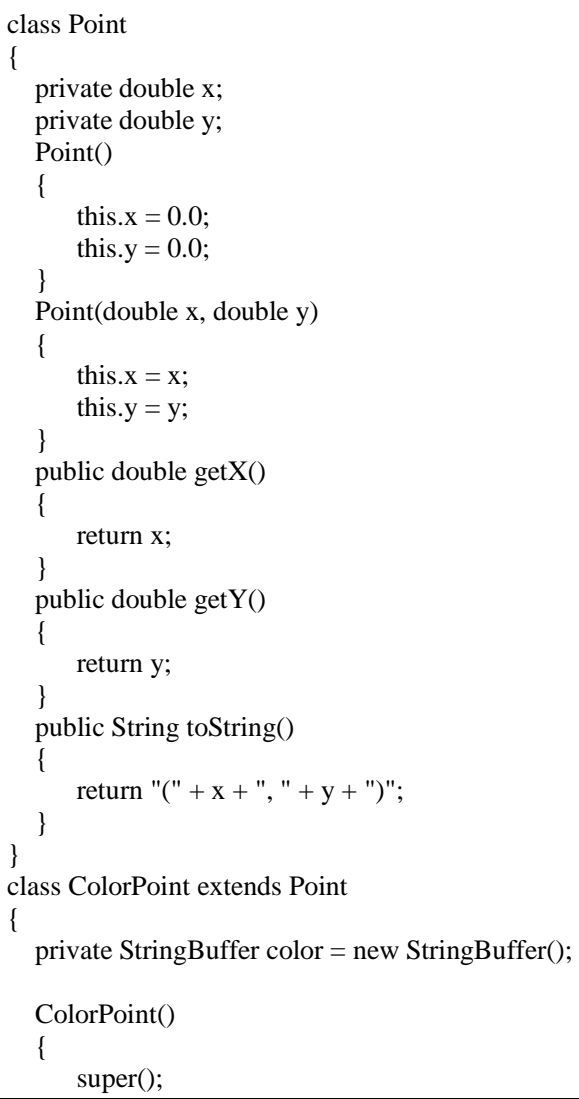 & $\begin{array}{l}\text { Nonterminal: PROGRAM } \\
\text { Nonterminal: CLASS_DCL } \\
\text { Terminal: Point } \\
\text { Nonterminal: CLASS_BODY } \\
\text { Nonterminal: FIELD_DCL } \\
\text { Nonterminal: PRIVATE } \\
\text { Nonterminal: DCL_SPEC } \\
\text { Nonterminal: DOUBLE_TYPE } \\
\text { Nonterminal: VAR_ITEM } \\
\text { Nonterminal: SIMPLE_VAR } \\
\text { Terminal: x } \\
\text { Nonterminal: FIELD_DCL } \\
\text { Nonterminal: PRIVATE } \\
\text { Nonterminal: DCL_SPEC } \\
\text { Nonterminal: DOUBLE_TYPE } \\
\text { Nonterminal: VAR_ITEM } \\
\text { Nonterminal: SIMPLE_VAR } \\
\text { Terminal: y } \\
\text {.. } \\
\text { Nonterminal: CLASS_DCL } \\
\text { Nonterminal: PUBLIC } \\
\text { Terminal: javaClassTest } \\
\text { Nonterminal: CLASS_BODY } \\
\text { Nonterminal: METHOD_DCL } \\
\text { Nonterminal: METHOD_HEAD } \\
\text { Nonterminal: PUBLIC } \\
\text { Nonterminal: STATIC } \\
\text { Nonterminal: METHOD_ITEM } \\
\text { Terminal: main } \\
\text { Nonterminal: PARAM_DCL_LIST } \\
\text { Nonterminal: PARAM_DCL } \\
\text { Nonterminal: DCL_SPEC } \\
\text { Nonterminal: ARRAY_TYPE } \\
\text { Nonterminal: SIMPLE_NAME } \\
\text { Terminal: String } \\
\text { Nonterminal: SIMPLE_VAR } \\
\text { Terminal: args } \\
\text { Nonterminal: BLOCK_BODY (start : 46 end : 52) }\end{array}$ \\
\hline
\end{tabular}




\begin{tabular}{|c|c|}
\hline 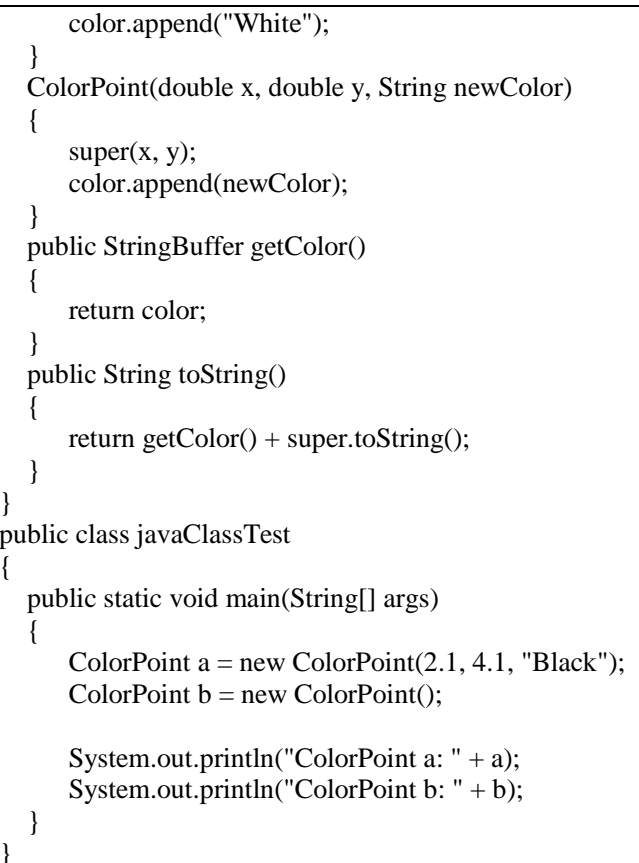 & $\begin{array}{l}\text { Nonterminal: LOCAL_VAR_DCL } \\
\text { Nonterminal: DCL_SPEC } \\
\text { Nonterminal: CLASS_INTERFACE_TYPE } \\
\text { Nonterminal: SIMPLE_NAME } \\
\text { Terminal: ColorPoint } \\
\text { Nonterminal: VAR_ITEM } \\
\text { Nonterminal: SIMPLE_VAR } \\
\text { Terminal: a } \\
\text {... }\end{array}$ \\
\hline
\end{tabular}

\section{Example 3. AST Generation for the Java Source Code}

Since the basic object information of each language has the same meaning, the AST of each object-oriented language can be expressed by extending the specific nodes having different differences in the language in the proposed AST structure.

Next, to investigate the source code complexity of the symbol generation structure, the cyclomatic complexity is calculated for the symbol table generation structure and the analysis structure for obtaining symbol information using SourceMonitor which is a complexity calculation tool. McCabe suggested cyclomatic complexity. If the complexity exceeds a certain value, it is difficult to manage the code. Therefore, it is recommended to keep the complexity below 10, and Microsoft's MSDN recommends keeping the complexity below 25 . Table 1 shows the bad fix probability according to the cyclomatic complexity[3]. The bad fix probability represents the odds of introducing an error while maintaining code.

Table 1. Bad Fix Probability According to the Cyclomatic Complexity

\begin{tabular}{|c|c|}
\hline Cyclomatic Complexity & Bad Fix Probability \\
\hline $1 \sim 10$ & $5 \%$ \\
\hline $20-30$ & $20 \%-40 \%$ \\
\hline $50-100$ & $40 \%$ \\
\hline$>100$ & $60 \%$ \\
\hline
\end{tabular}

Next, Figure 12 shows the result of analyzing the source code complexity of the existing symbol generation structure and the proposed symbol generation structure. The existing symbol generation structure has a complexity of 27.5 on average, another error of $20-40 \%$, and a probability of error of $60 \%$ in routines with a maximum of 405 complexity. The average complexity of the proposed symbol generation structure is 1.94 , and the error probability is less than $5 \%$. The maximum complexity is 6 , so that the probability of error occurrence is also less than $5 \%$ for the most complex routines. The generated code has a block depth of up to 9 and a mean block depth of 4.77, whereas the proposed symbol generation structure has a maximum block depth of 4 and an average block depth of 1.53. So, it can be seen that the routine is simplified. As a result of 
analysis, it is confirmed that the complexity and the block depth of the proposed generation structure are greatly improved compared with the existing generation structure.

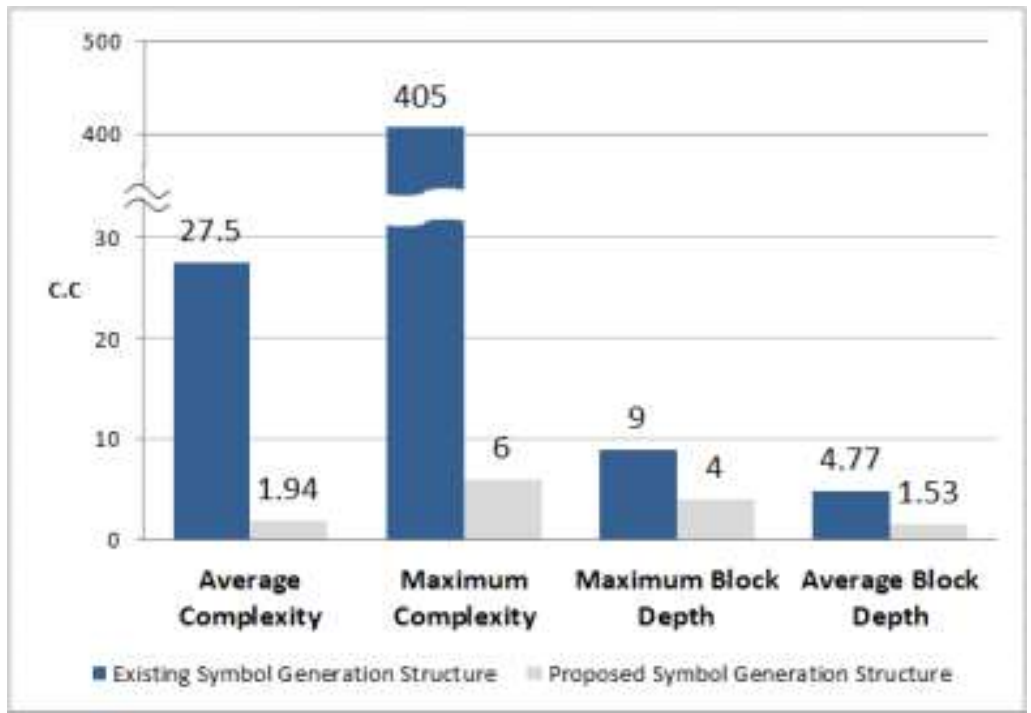

\section{Figure 12. Source Code Complexity Analysis Result of Existing Symbol Generation Structure and Proposed Symbol Generation Structure}

Figure 13 shows the results of the cyclomatic complexity analysis of the intermediate language generator module that generates the intermediate language by referring to the existing symbol table and the improved symbol table. As a result of investigation of the complexity of the routine for generating the intermediate language by referring to the symbol table, the intermediate language generation structure using the existing symbol table has a complexity of 11.5 on average and another error of $20-40 \%$. Also, the routine with the maximum complexity has a value of 361 and has a probability of occurrence of error of $60 \%$. In contrast, the intermediate language generation structure using the proposed symbol table exhibits an average complexity of 1.67 , the probability of error occurrence is less than $5 \%$, and the routine with the maximum complexity has a complexity of 33 , which shows $90 \%$ improvement compared to the existing structure.

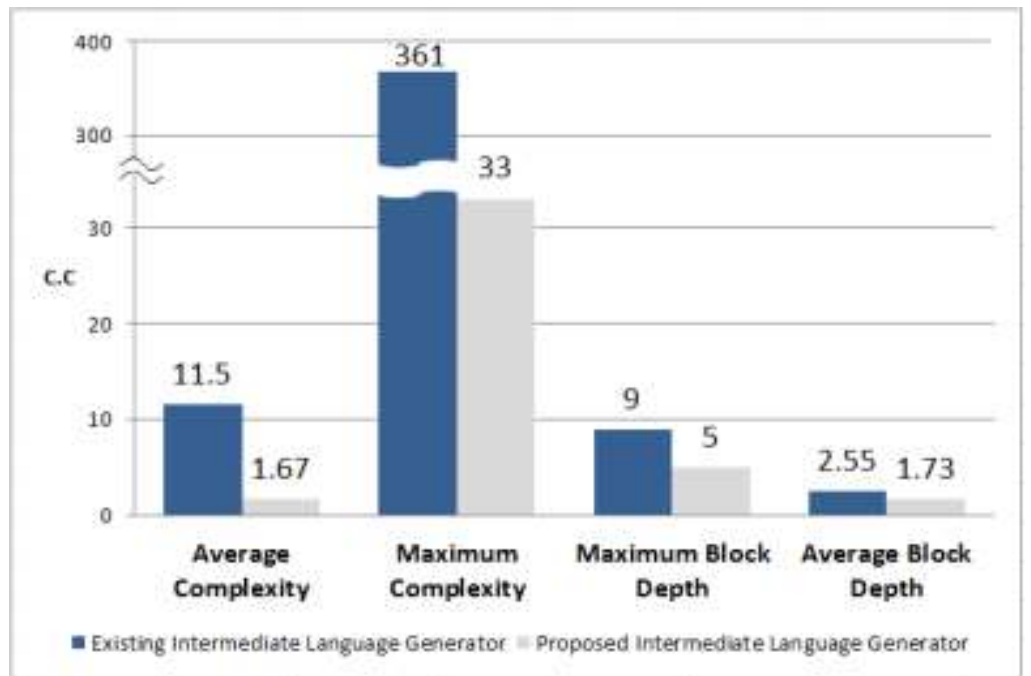

Figure 13. Source Code Complexity Analysis Result of the Intermediate Language Generation Structure using the Existing Symbol Table and the Proposed Symbol Table 
The generated code has a block depth of up to 9 and a mean block depth of 2.55, while the proposed structure has a maximum block depth of 5 and an average block depth of 1.73. It can be seen that the routine for generating the intermediate language is simplified by referring to the symbols. As a result of the analysis, it is confirmed that the complexity and the block depth of the intermediate language generation structure using the proposed symbol table are greatly improved when using the existing symbol table. As a result of the analysis, it can be seen that the average and maximum complexity are improved when the existing symbol table and the proposed symbol table are used in both the symbol generation structure and the intermediate language generation structure.

\section{Conclusions}

Since a conventional symbol table can't represent symbols in one table, symbol attributes are divided and stored in a plurality of tables. A module referring to a symbol table refers to each of a plurality of tables, collects attributes, and had to complete symbol information again. In addition, in the case of an abstract syntax tree used to express the characteristics of a language and to generate symbols, since the language features are expressed through simple nodes, the abstract syntax tree has to be newly designed to add linguistic features even if the basic object-oriented language is the same, and new symbol generation routines and symbol table reference routines for the languages have to be added. Such an abstract syntax tree structure and a symbol expression scheme increase the complexity of a routine for generating symbols and a routine for referring to symbols.

In order to solve this problem, this paper has designed hierarchical structure of AST nodes by applying object orientation principle and Visitor pattern and improved the symbol table by hierarchical structure of symbols. With the improved AST structure, the symbol generation routine analyzes the nodes of the AST without additional conditional statements, and collects symbol information and makes it possible to easily add additional linguistic features into the hierarchical subclass form. The complexity of the symbol generation routine can be reduced because the symbols are assembled into the symbol table by the node traversal and inserted into the symbol table using the object orientation principle. Since the improved symbol table stores symbols designed in a hierarchical structure, symbol information can be directly obtained from the symbol reference routines. Therefore, a module using a symbol table simplifies the process of obtaining symbol information, and even if the information of the symbol is complicated, the additional complexity is not needed, and the complexity becomes simpler than the conventional one.

\section{Acknowledgement}

This Research was supported by Seokyeong University in 2015.

\section{References}

[1] S. C. Dewhurst, "Flexible symbol table structures for compiling C++," Software - Practice and Experience, vol. 17, (1987), pp. 503-512.

[2] R. P. Cook, T. J. Leblanc, "Symbol table abstraction to implement languages with Explicit Scope Control," IEEE Transactions on Software Engineering, vol. 9, (1983), p. 8.

[3] S. C. Dewhurst, "Flexible symbol table structures for compiling C++," Software: Practice and Experience, vol. 17, no. (1987), pp. 503-512.

[4] M. Gallego-Carrillo, F. Gortázar-Bellas, J. Urquiza-Fuentes and J.Á. VelázquezIturbide, "SOTA: A visualization tool for symbol tables," ACM SIGCSE Bulletin, vol. 37, (2005), p. 385

[5] J. F. Power, B. A. Malloy, "Symbol Table Construction and Name Lookup in ISO C++," 37th International Conference on Technology of Object-Oriented Languages and Systems, TOOLS 2000, (2000), p. 57.

[6] Y. S. Son, Y. S. Lee, "The Reverse Translator for Symbol Table Verification in Objective C compiler on Smart Cross Platform," The Asian International Journal of Life Sciences, vol. 11, (2015), p. 625.

[7] Y. S. Lee, Y. S. Son, "The Reverse Translator for Reconstruction of the Declaration Part from Symbol Tables of the C++ Compiler," Information-an International Interdisciplinary Journal, vol. 16, (2013), p. 2319. 
[8] Y. S. Lee, Y. S. Son, "A Study on Verification and Analysis of Symbol Tables for Development of the C++ Compiler," International Journal of Multimedia and Ubiquitous Engineering, SERSC, vol. 7, (2012), p. 175.

[9] Y. S. Lee, Y. K. Kim, H. J. Kwon, "Design and Implementation of the Decompiler for Virtual Machine Code of the C++ Compiler in the Ubiquitous Game Platform," LNCS, vol. 4413, (2007), p. 511

[10] H. J. Kwon, Y. K. Kim, J. K. Park, Y. S. Lee, "Design and Implementation of a Detranslator for Verification and Analysis of Symbol Tables in an ANSI C Compiler," The 2006 International Conference on Multimedia, Information Technology and its Applications (MITA2006), (2006), Dalian, China

[11] Y.S. Lee, "The Virtual Machine Technology for Embedded Systems," Journal of the Korea Multimedia Society, vol. 6, (2002), p. 36.

[12] R. C. Martine, Agile Principles, Patterns, and Practices in C\#, Pearson Education, (2006).

[13] E. Freeman, Elisabeth Freeman, K. Sierra, B. Bates, Head First Design Patterns, O’Reilly, (2004).

[14] L. M. Laird, M. C. Brennan, Software Measurement and Estimation: A Practical Approach, WILEY, (2006)

[15] M. H. Halstead,, Elements of Software Science, New York: Elsevier North-Holland, (1977).

[16] SourceMonitor, http://www.campwoodsw.com/sourcemonitor.html

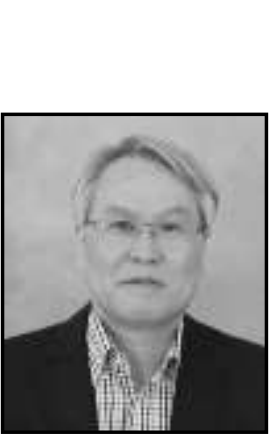

\section{Author}

Yangsun Lee, He received the B.S. degree from the Dept. of Computer Science, Dongguk University, Seoul, Korea, in 1985, and M.S. and Ph.D. degrees from Dept. of Computer Engineering, Dongguk University, Seoul, Korea in 1987 and 2003, respectively. He was a Manager of the Computer Center, Seokyeong University from 1996-2000, a Director of Korea Multimedia Society from 20042005, a General Director of Korea Multimedia Society from 20052006, a Vice President of Korea Multimedia Society in 2009, and a Senior Vice President of Korea Multimedia Society in 2015-2016. Also, he was a Director of Korea Information Processing Society from 2006-2014 and a President of a Society for the Study of Game at Korea Information Processing Society from 2006-2010. And, he was a Director of HSST from 2014-2016. Currently, he is a Professor of Dept. of Computer Engineering, Seokyeong University, Seoul, Korea. His research areas include smart system solutions, programming languages, and embedded systems. 
International Journal of Database Theory and Application Vol.10, No.7 (2017) 